\title{
Predicted elastic constants and critical layer thicknesses for cubic phase AIN, GaN, and InN on $\beta$-SiC
}

\author{
M. E. Sherwin \\ Department of Electrical Engineering and Computer Science, University of Michigan, Ann Arbor, \\ Michigan 48109-2122
}

\author{
T. J. Drummond \\ Sandia National Laboratories, P.O. Box 5800, Albuquerque, New Mexico 87185
}

(Received 25 October 1990; accepted for publication 21 March 1991)

\begin{abstract}
Elastic constants for zinc-blende AlN, GaN, and InN have been estimated from the elastic constants of the wurtzite phase. This has been accomplished by recognizing that the crystal structures of the wurtzite and zinc-blende phases are related by a simple rotation. This rotation was then applied to the elastic constants and a least-squares fit is used to match the results. Using the zinc-blende elastic constants the critical thickness of the nitrides on $\beta$-SiC substrates was calculated. The critical thickness of a single overlayer of A1N was calculated to be $14.1 \mathrm{~nm}$, and for $\mathrm{GaN}$ the critical thickness was found to be $0.7 \mathrm{~nm}$. In the elastic continuum model used there was no solution for the critical thickness of InN.
\end{abstract}

Aluminum, gallium, and indium nitrides are of potential interest as optical devices. Most of the work done in the past with these materials has been on sapphire substrates, growing the nitride in a wurtzite crystal form. Recently, it has been demonstrated that the cubic phase of $\mathrm{GaN}$, and possibly AlN, will grow on $\beta$-SiC or even GaAs substrates. ${ }^{1,2}$ It is expected that cubic phase nitrides will have unique electrical properties. Certainly the relatively close lattice match to $\beta$-SiC makes them interesting.

Prediction of the critical layer thickness of pseudomorphic cubic nitride films on $\beta$-SiC substrates requires a knowledge of the lattice parameter and the elastic constants of the nitride overlayer. Since only a few small samples of cubic phase nitrides have been fabricated, very little is known about any of the electronic, optical, or mechanical properties thereof. In this paper, the measured elastic constants of all three nitrides in the wurtzite phase are collected for the first time in a single report and used to predict the elastic properties of the cubic phase crystals. These results are then used to calculate critical layer thicknesses for cubic phase AlN, GaN, and InN on $\beta$-SiC.

The elastic properties of the wurtzite and zinc-blende crystal structures may be correlated by observing that the two structures are identical through the second nearest neighbor. It has also been recognized that it is possible to construct both lattices from a simple (zinc-blende) tetrahedral building block. ${ }^{3}$ Martin has developed a procedure for relating the elastic constants of a zinc-blende crystal to its wurtzite counterpart. ${ }^{4}$ Martin's method consists of applying two inequivalent rotations to the fourth-rank elastic tensor of the zinc-blende crystal defining two trigonal elastic tensors corresponding to the two inequivalent tetrahedral building blocks which, when superimposed, result in the wurtzite structure. Minimizing the internal strain of the resulting system results in a simple relationship between the elastic constants of the zinc-blende crystal and the wurtzite crystal. In writing out this relationship, the fourth-rank tensors are reduced to simple vectors with the following definitions:

$$
C_{1}^{\mathrm{ZB}}=C_{11}^{\mathrm{ZB}}, \quad C_{2}^{\mathrm{ZB}}=C_{12}^{\mathrm{ZB}}, \quad C_{3}^{\mathrm{ZB}}=C_{44}^{\mathrm{ZB}}
$$

and

$$
\begin{array}{lll}
C_{1}^{\mathrm{WZ}}=C_{11}^{\mathrm{WZ}}, & C_{2}^{\mathrm{WZ}}=C_{33}^{\mathrm{WZ}}, & C_{3}^{\mathrm{WZ}}=C_{12}^{\mathrm{WZ}}, \\
C_{4}^{\mathrm{WZ}}=C_{13}^{\mathrm{WZ}}, & C_{5}^{\mathrm{WZ}}=C_{44}^{\mathrm{WZ}}, & C_{6}^{\mathrm{WZ}}=C_{66}^{\mathrm{WZ}} .
\end{array}
$$

The equations relating the two vectors are

$$
C_{i}^{\mathrm{WZ}}=\bar{C}_{i}^{\mathrm{WZ}}-D_{i}, \quad i=1, \ldots, 6
$$

where

$$
\begin{aligned}
& \bar{C}_{i}^{\mathrm{WZ}}=\sum_{j=0}^{3} P_{i j} C_{j}^{\mathrm{ZB}} \\
& \Delta=\sum_{j=0}^{3} Q_{j} C_{j}^{\mathrm{ZB}} \\
& D=\left[\frac{\Delta^{2}}{\bar{C}_{5}^{\mathrm{WZ}}}, 0, \frac{-\Delta^{2}}{\bar{C}_{5}^{\mathrm{WZ}}}, 0, \frac{\Delta^{2}}{\bar{C}_{6}^{\mathrm{WZ}}}, \frac{\Delta^{2}}{\bar{C}_{5}^{\mathrm{WZ}}}\right]^{T}, \\
& P=\frac{1}{6}\left[\begin{array}{rrr}
3 & 3 & 6 \\
2 & 4 & 8 \\
1 & 5 & -2 \\
2 & 4 & -4 \\
2 & -2 & 2 \\
1 & -1 & 4
\end{array}\right] \text {, } \\
& Q=\sqrt{2} / 6\left[\begin{array}{lll}
1 & -1 & -2
\end{array}\right] \text {. }
\end{aligned}
$$

As written, these equations will produce the wurtzite elastic constants from known zinc-blende data. ${ }^{5}$ To perform the opposite and desired operation, it was necessary to use a least-squares fitting routine to find the zinc-blende constants that provided the closest reverse transformation to the known wurtzite elastic constants. Martin demonstrated the method for the test case of $\mathrm{ZnS}$ for which experimental data were available for both crystal structures. Although 
TABLE I. Wurtzite and zinc-blende elastic constants for AIN, GaN, and InN. All units arc $10^{11}$ dyncs $/ \mathrm{cm}^{2}$.

\begin{tabular}{|c|c|c|c|c|c|c|}
\hline & \multicolumn{2}{|c|}{ AlN } & \multicolumn{2}{|c|}{$\mathrm{GaN}$} & \multicolumn{2}{|c|}{$\operatorname{InN}$} \\
\hline & WZ & $\mathrm{ZB}$ & WZ & ZB & WZ & ZB \\
\hline$C_{11}$ & 34.5 & 32.8 & 29.6 & 26.4 & 19.0 & 17.2 \\
\hline$C_{33}$ & 39.5 & & 26.7 & & 18.2 & \\
\hline$C_{12}$ & 12.5 & 13.9 & 13.0 & 15.3 & 10.4 & 11.9 \\
\hline$C_{\mathrm{I} 3}$ & 12.0 & & 15.8 & & 12.1 & \\
\hline$C_{44}$ & 11.8 & 13.3 & 2.4 & 6.8 & 1.0 & 3.7 \\
\hline$C_{66}$ & 11.0 & & 8.3 & & 4.3 & \\
\hline Ref. & 11 & & 12 & & 13 & \\
\hline
\end{tabular}

the accuracy of the experimental elastic constants is not perfect a correspondence of $6 \%$ or better in the wurtzite to zinc-blende transformation was obtained. The known wurtzite and calculated zinc-blende elastic constants of the III-N nitrides are summarized in Table I.

To compare these results to existing theoretical estimations of the elastic properties of cubic III-N crystals we note that Van Camp et al. ${ }^{6,7}$ have calculated the bulk modulus of $\mathrm{GaN}$ and InN using the local density approximation with norm-conserving nonlocal pseudopotentials. They report the bulk moduli of $\mathrm{GaN}$ and $\mathrm{InN}$ to be 158 and $155 \mathrm{GPa}$, respectively. The bulk modulus of zincblende crystals may be derived from the elastic constants as $B=\left(c_{11}+2 c_{12}\right) / 3$. The calculated values of the bulk modulus (in $\mathrm{GPa}$ ) for AlN, GaN, and InN are 202, 185, and 136 , respectively. These values agree to within $20 \%$ of the calculations of Van Camp et al. Although the agreement is not very good Van Camp observes that the pseudopotential results for nitride compounds is expected to be relatively poor as compared to phosphide and arsenide compounds due to the small size of the nitrogen atom.

In calculating the critical layer thicknesses for the three nitride compounds on a $\beta$-SiC substrate, the force balance method of Matthews and Blakeslee ${ }^{8}$ is used. This method balances the line tension present on a dislocation against the glide force present in a strained film. For a single overlayer with only one interface, the equation for the critical film thickness is given by

$$
h=\frac{a(1-v / 4)}{4 \sqrt{2} \pi(1+v) \epsilon}\left[\ln \left(\frac{\sqrt{2} h}{a}\right)+\theta\right]
$$

where $h$ is the film thickness, $a$ is the substrate lattice constant, $\epsilon=\left(a_{\text {nitride }}-a_{\mathrm{SiC}}\right) / a_{\text {nitride }}$ is the strain present in the film, and $\theta \approx 1$ represents the dislocation core energy. There are several assumptions that must be made for this equation to be valid in the following analysis. To relieve strain effectively the $a$ dislocation must run along the interface between the substrate and the overlayer. In the present case, the interface is between a IV-IV zinc blende and III-V zinc blende semiconductor. The nature of such a dislocation is problematic and it must be assumed that it does not significantly modify our analysis. Second, for large mismatches the elastic constants themselves are modified by lattice distortion as is the dislocation core energy.
TABLE II. Estimated cubic lattice constants, strain relative to $\beta$-SiC, Poisson's ratio, and pseudomorphic critical layer thickness for cubic nitrides on $\beta$-SiC. In the case of InN no solution exists within the continuum elastic approximation for an overlayer on $\beta$-SiC.

\begin{tabular}{lcccc}
\hline \hline & $a(\mathrm{~nm})$ & $\epsilon(\%)$ & $v$ & $h(\mathrm{~nm})$ \\
\hline AlN & 0.438 & 0.6 & 0.297 & 14.1 \\
GaN & 0.454 & 4.1 & 0.366 & 0.7 \\
InN & 0.503 & 15.3 & 0.408 & $\cdots$ \\
\hline \hline
\end{tabular}

These two effects appear to compensate one another, at least in the case of III-V/III-V strained layer systems. ${ }^{9}$ In the present context it is expected that the results of the critical layer thickness calculations are accurate to within a factor of two.

The lattice constant of $\beta-\mathrm{SiC}$ is $0.4359 \mathrm{~nm}$. In calculating the strain in the nitride film, the zinc-blende lattice constants were derived from the wurtzite data in Refs. $10-13$. For an ideal wurtzite structure, $c / a=1.633$ and the equivalent cubic lattice constant is related to the wurtzite data by the relation $a_{\mathrm{ZB}}=(\sqrt{3} / 2) c_{\mathrm{WZ}}$. Beyond this simple relationship, experimental data are available for $\mathrm{GaN}{ }^{2}$ These data indicate that the nonideal wurtzite structure has a scaling factor to the zinc-blende structure other than $(\sqrt{3} / 2)=0.8660$. This scaling factor was found to be 0.881 . As a first-order approximation, this scaling factor was also used for AIN and InN. Poisson's ratio is obtained by first transforming the elastic constants to compliances $\left(s_{i j}\right)$ then $v=-s_{12} / s_{11}$. Values of $a, \epsilon$, and $v$ for the three nitrides are listed in Table II. Inserting these values into the Matthews and Blakeslee equation gives the following critical layer thicknesses: $h(\mathrm{AlN})=14.1 \mathrm{~nm}$ and $h(\mathrm{GaN})=0.7 \mathrm{~nm}$. No solution was found for InN within the elastic continuum model.

Although these thicknesses appear to place considerable limitations on device structures, it appears that it may still be possible to alleviate the strain in thick nitride films without introducing dislocations. Paisley ${ }^{1}$ has noticed a thin "amorphous" region between a $\beta$-SiC substrate and an overgrown $\mathrm{GaN}$ film. After this amorphous region the GaN film grows in an undislocated cubic phase. Instead of an amorphous region, we speculate that this region could be due to a unique hexagonal stacking sequence, since it is possible that $\mathrm{GaN}$ can exist in numerous polytypes as is true of SiC. It is possible that some of the strain present is accommodated by this stacking sequence. Although this phenomenon may preclude the growth of pseudomorphic thin films it seems to offer promise for growing thick films of cubic nitrides.

The elastic constants of wurtzite phase AlN, GaN, and InN have been collected. From these data a set of elastic constants for the zinc-blende phases has been estimated. Using the calculated values of Poisson's ratio and the lattice constants, critical layer thicknesses were calculated for cubic phase growth on $\beta$-SiC substrates. Critical layer thicknesses of 14.1 and $0.7 \mathrm{~nm}$ were derived for AIN and $\mathrm{GaN}$, respectively. In the case of InN, the lattice mismatch is estimated to be sufficiently large that no solution for the 
critical layer thickness exists within the elastic continuum model.

The authors would like to thank Dr. B.W. Dodson for his insights as to the applicability of the Matthews and Blakeslee theory. This work was primarily supported by the U.S. Army Research Office, URI Program, Contract No. DAAL03-86-K-0007. The work at Sandia was supported by the U.S. Dept. of Energy under Contract No. DE-AC04-76DP00789.

${ }^{1}$ M. J. Paisley, Z. Sitar, J. B. Posthill, and R. F. Davis, J. Vac. Sci. Technol. A 7, 701 (1989).

${ }^{2}$ M. Mizuta, S. Fujieda, Y. Matsumoto, and T. Kawamura, Jpn. J. Appl. Phys. 25, L945 (1986).

${ }^{3}$ F. N. H. Robinson, Phys. Lett. 26A, 435 (1968).

${ }^{4}$ R. M. Martin, Phys. Rev. B 6, 4546 (1972).
${ }^{5}$ In the paper by Martin, there was a typographical error in the definition of the $Q$ matrix.

${ }^{6}$ P. E. Van Camp, V. E. Van Doren, and J. T. Devreese, Phys. Rev. B 38, 9906 (1988).

${ }^{7}$ P. E. Van Camp, V. E. Van Doren, and J. T. Devreese, Phys. Rev. B 41, 1598 (1990).

${ }^{8}$ J. W. Matthcws and A. E. Blakeslee, J. Cryst. Growth 27, 118 (1974). ${ }^{9}$ T. G. Andersson, Z. G. Chen, V. K. Kulakovskii, A. Uddin, and J. T. Vallin, Appl. Phys. Lett. 51, 752 (1987).

${ }^{10}$ Landholt and Börnstein, Group III: Crystal and Solid State Physics (Springer, Berlin-Heidelberg, 1984), Vol. 18.

${ }^{11} \mathrm{~K}$. Tsubouchi and N. Mikoshiba, IEEE Trans. Sonics Ultrason. SU-32, 634 (1985).

${ }^{12}$ V. A. Savastenko and A. U. Sheleg, Phys. Status Solidi A 48, K135 (1978).

${ }^{13}$ A. U. Sheleg and V. A. Savastenko, Inorg. Mater. (USA) 15, 1257 (1979). 